\title{
Design and analysis of the flange-bolted joint with respect to required tightness and strength
}

https://doi.org/10.1515/eng-2019-0031

Received February 12, 2019; accepted April 8, 2019

\begin{abstract}
The paper presents the method of design and strength analysis of the flange-gasketed-bolted joint. In the first part, analytical calculations were carried out. Their purpose was to determine the assembly torque of nuts to achieve the desired tightness. The flanged joint designated as DN100 PN40 with two different gaskets was taken into consideration. The analytical calculations were performed in accordance with the algorithm included in PN EN 1591-1. In the further step, the numerical calculations were carried out to support the analytical results. The outcome of these calculations were maps of the contact stress distribution on the gasket surface, estimation of safety factors of individual joint elements as well as determination of the flange rotation. Data from the numerical calculations were compared with the analytical results, which confirmed their satisfactory compliance. In the last stage, the experimental tests of the joint were carried out. The main results of the test were the measured values of the leakage level, tightening force of the bolt and estimated safety factors. An indirect result of the experimental measurements were maps of the stress distribution on the contact surface of the gasket, which were determined by means of the measuring film. Ultimately, based on the experimental results, it was found that the proposed analytical method of calculation and simulation of the joint by means of the finite element method was a very good tool for the design of the joint at the required tightness level.
\end{abstract}

\footnotetext{
Przemysław Jaszak: Wroclaw University of Science and Technology, Department of Mechanical and Power Engineering, Wybrzeże Wyspiańskiego Street 27, 50-370 Wrocław, Poland,

E-mail: przemyslaw.jaszak@pwr.edu.pl

^Corresponding Author: Konrad Adamek: Gambit Lubawka

Sp. z o. o. Lubawka Wojska Polskiego 16 58-420, Poland,

E-mail: konrad.adamek@gambitgl.pl
}

\section{Introduction}

A flange-bolted joint is one of the basic nodes of the pipeline system enabling connection of singular segments of pipes into more complex sections or joining measuring and processing devices and machines, such as flowmeters, pumps, fans and pressure vessels. It is estimated that in a medium-sized refinery there are approximately 100,000 such connections. Due to the fact that it is a demountable joint, it is a point where leakage is inevitable. This leakage should be determined at the design stage [1] and, if possible, strictly controlled during the assembly and operating condition of the pipeline systems [2, 3]. Most of the pipeline installations transport substances, which due to uncontrolled leakage can contribute to major disasters $[4,5]$. Depending on the type of transported medium it may cause a hazard of fire, environmental contamination, thus it entails exposure to a loss of health and life of people or animals [6].

A design of such connections mainly depends on the selection of a proper gasket/seal and its appropriate assemble by adequate loading. The basic design data are: the nominal diameter of the pipeline, temperature, pressure and the type of transported medium. Additional data are external loads, their alterations in time, required tightness and durability of the joint. Over the decades, many computational algorithms have been developed to support the design of the flange-bolted joint in respect to the desired tightness. In most cases, the procedures refer to the design of a flange-bolted joint with a circular gasket, and the results of the calculation were mainly in the form of the tightening force of the bolt or torque at which the nuts should be tightened to ensure proper tightness [7]. The calculation codes [8], [9] were based on a one-dimensional joint model in which the gasket, bolts and flanges are not deformed. The most precise approach to the design of such connections is in the algorithm according to [1]. The computational model considers, among others, interactions of the flange-gasket-bolt, their elastic-plastic behavior as well as a creep and relaxation of the gasket subjected to elevated temperature. Additionally, the external loads in the form of forces and bending moments, thermal expansion of the 
components as well as friction conditions on the mating surfaces are taken into account. The algorithm considers two basic load states:

I-0 - in service state (assembly condition),

I-1 - operating state (impact of the internal pressure and temperature as well as external load acting on the joint).

Necessary design data, in the form of the material properties of the gasket, were obtained from the experimental tests in accordance with [10].

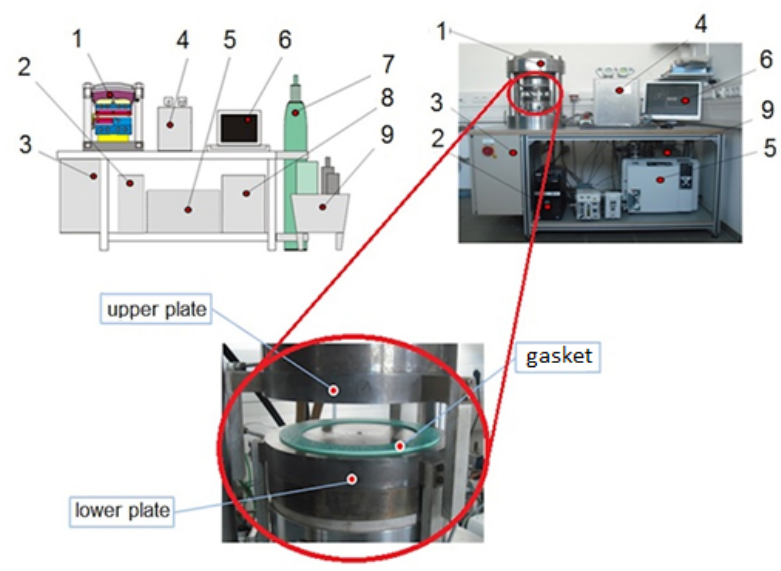

Figure 1: Test rig to determine materials properties of gasket; 1 hydraulic press, 2 - computer, 3 - control board, 4 - differential pressure units, 5- helium detector, 6 - screen, 7 - helium reservoir, 8 - cooling unit of the hydraulic press, 9 - oil pump of the hydraulic press

These tests were carried out on a special device (see Fig. 1) simulating a load of the gasket in a given temperature and pressure of the sealed medium (usually helium). The direct results of the tests are the deformation characteristics or tightness characteristics of the gaskets. Based on such data, calculation coefficients were determined. These coefficients, among others, were: elastic modulus, coefficient of friction, allowable contact stress, minimum contact stresses providing adequate tightness. In a properly designed joint (besides achieving desired tightness), an important aspect is to maintain the stress in particular joint elements under a permissible value. A safe condition is distinguished by means of a safety factor, which indirectly describes the state of load of a particular joint element. In general, this safety factor determines the ratio of the current value of force or stress in a given element over the value of the limit force or permissible stress. To fulfill a failure-free operation the inequalities (1) for all the abovementioned load states I-0, I-1 had to be met.

$$
\varphi=\frac{F_{A}}{F_{\text {Lim }}}=\frac{f_{A}}{f_{\text {Lim }}} \leq 1
$$

Where:

$F_{A}, F_{\text {Lim }}$ - the actual force applied to the elements and limit force causes failure of the elements respectively,

$f_{A}, f_{\text {Lim }}$ - the actual stress in elements and limit stress (equals permissible stress) respectively.

A certain complement to the analytical calculations and their verification may be collaterally conducted using numerical calculations, based on the finite element method (FEM). The analysis of such issues has been presented, among others, in [11-17]. The geometric model can be constructed, on the basis of a 2D (axi-symmetricity) or 3-D [18] analysis of the whole or part of the joint. The main advantages of numerical calculations are primarily:

- determination of the contact stress on the surface of the gasket,

- determination of the maximum equivalent stress of the flanges, bolts and gasket,

- determination of the influence of thermal and external loads on the joint deformation level.

The data in the form of the material properties of the gasket and assembly loads, necessary to prepare a computational model, were obtained by means of the above-mentioned analytical calculations and experimental tests.

\section{Aim and scope of the work}

The purpose of the work was to design a flange-bolted joint with a gasket for two strictly defined tightness classes. In the project, as basic tools, the analytical calculations (according to the procedure described in [1]) and the finite element method were used. The analytical calculations were mainly used to determine the in service bolt tension and estimate the safety factors of the bolt, flange and the gasket. In the last stage, experimental tests were carried out to determine the actual level of tightness and verify the analytical and numerical calculations.

\section{Design data}

The key data were the characteristic dimensions of the PN40 DN100 flange-bolted joint (see Fig. ??) and the values listed in Table 1.

As a gasket material, two types of $2 \mathrm{~mm}$ thick fibrouselastomer material were selected. The material marked as FG_1 was a composite made from a mixture of aramid and mineral fibers and NBR rubber as a binder. In addition, the material was internally reinforced with a perforated mesh 
Table 1: Design data of the PN40 DN 100 flange-bolted joint

\begin{tabular}{|c|c|c|c|c|}
\hline No. & Parameter & Symbol & Value & Unit \\
\hline \multicolumn{5}{|c|}{ Operating parameters } \\
\hline 1 & Pressure & $\mathrm{p}$ & 40 & bar \\
\hline 2 & Fluid temperature & $T$ & 20 & $\mathrm{C}$ \\
\hline \multicolumn{5}{|c|}{ Flanges } \\
\hline 3 & External diameter & D & 275 & $\mathrm{~mm}$ \\
\hline 4 & Pitch Circle Diameter & $D_{b}$ & 210 & $\mathrm{~mm}$ \\
\hline 5 & Pipe diameter & $D_{p}$ & 100 & $\mathrm{~mm}$ \\
\hline 6 & Flange thickness & $\mathrm{t}$ & 38.5 & $\mathrm{~mm}$ \\
\hline 7 & Permissible stress & $f_{F}$ & 192 & $\mathrm{MPa}$ \\
\hline \multicolumn{5}{|c|}{ Gasket } \\
\hline 8 & Outer diameter & $\mathrm{d}_{\mathrm{o}}$ & 152 & $\mathrm{~mm}$ \\
\hline 9 & Inner diameter & $\mathrm{d}_{\mathrm{i}}$ & 112 & $\mathrm{~mm}$ \\
\hline 10 & Gasket thickness & $t_{g}$ & 2 & $\mathrm{~mm}$ \\
\hline 11 & Permissible stress & $f_{G}$ & 220 & $\mathrm{MPa}$ \\
\hline 12 & Material & \multicolumn{3}{|c|}{ FG 1/ FG 2} \\
\hline \multicolumn{5}{|c|}{ Bolts } \\
\hline 13 & Number of bolts & $\mathrm{n}_{\mathrm{b}}$ & 8 & - \\
\hline 14 & Bolt strength & $f_{B}$ & 500 & $\mathrm{MPa}$ \\
\hline 15 & Bolt diameter & M & 24 & $\mathrm{~mm}$ \\
\hline 16 & Bolt overall length & $l_{0}$ & 100 & $\mathrm{~mm}$ \\
\hline 17 & Bolt grip length & $\lg$ & 80 & $\mathrm{~mm}$ \\
\hline 18 & Permissible stress & $f_{B}$ & 500 & $\mathrm{MPa}$ \\
\hline
\end{tabular}

made of $0.25 \mathrm{~mm}$ thick stainless steel. The second material was a composite designated as FG_2 consisting of aramid, graphite and mineral fibers as well as SBR rubber as a binder. Both materials were characterized with high resistance to liquid and gas fuel and a wide range of load application from vacuum pressure to $100 \mathrm{bar}$, and temperature from -65 to $250^{\circ} \mathrm{C}$. Two tightness classes were assumed in the calculations: L1.0 and L.0.1, which corresponded to leakage levels: $1.0 \mathrm{mg} / \mathrm{s} \cdot \mathrm{m}$ and $0.1 \mathrm{mg} / \mathrm{s} \cdot \mathrm{m}$ related to the mean gasket perimeter. The coefficients of the gasket material necessary for the calculations process were obtained by the experimental tests [10], whose main results were characteristics describing the leakage and deformation level as a function of the contact stress. These characteristics were shown in Figures from 3 to 6 . The operating temperature of the design was $20^{\circ} \mathrm{C}$ and the pressure was 40 bar (4 MPa).

\section{Analytical calculations}

The purpose of analytical calculations was to determine the value of tightening torque of the nut in service state resulting from the force ensuring the proper joint tightness. Indirect results were, the estimation of the safety factors of the flange, bolt and gasket, as well as determination of the flange rotation angle. The basic formulas from the computational algorithm [1] were presented from (2) to (8).

Bolt force in service state:

$$
F_{B 0}=F_{G 0}+F_{R 0}
$$

Where $F_{G 0}$ is the gasket force which is found in the iterative calculation and $\mathrm{F}_{R 0}$ is an external load.

Bolt force in operating state:

$$
F_{B 1}=F_{G 1}+\left(F_{Q 1}+F_{R 1}\right)
$$



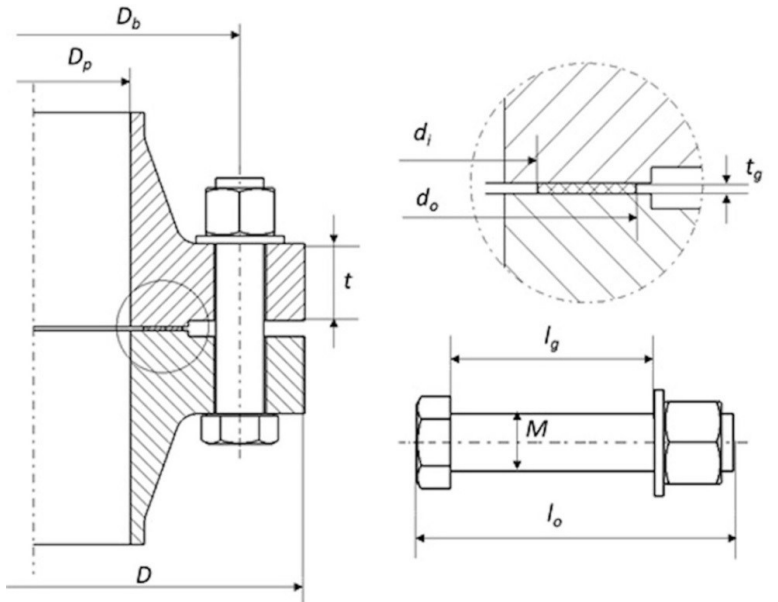

Figure 2: The characteristic dimension of the flange-bolted joint

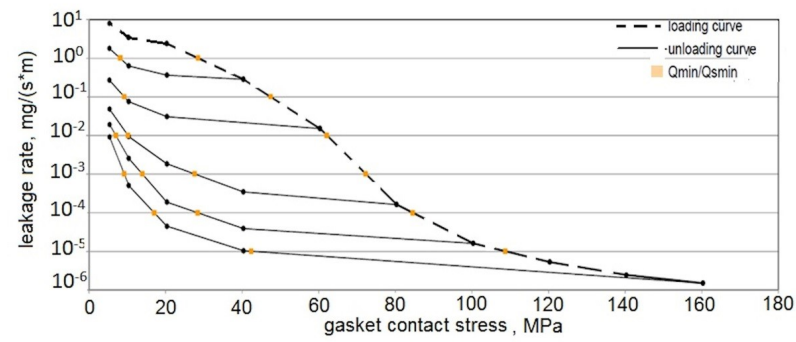

Figure 3: The characteristic describing leakage rate vs applied contact stress of the FG_1 gasket

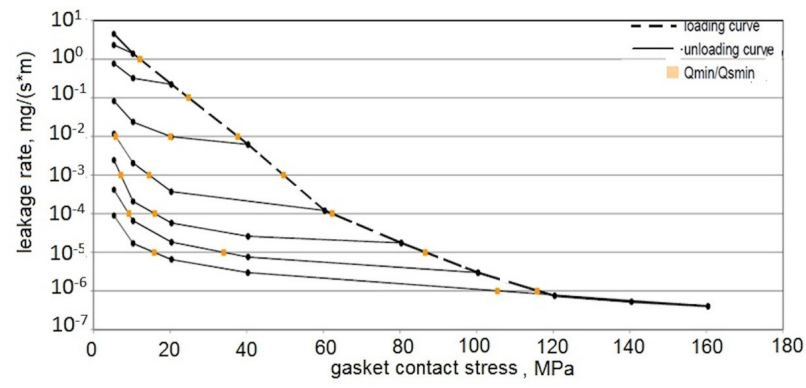

Figure 4: The characteristic of FG_2 gasket describing the leakage level as a function of contact stress

Where:

$F_{Q}-$ is a hydrostatic force resulting from fluid pressure.

Each parameter indexed as $\mathbf{1}$ means in service state, whereas parameters indexed as $\mathbf{0}$ means in operating state.

Bolt load ratio (safety factor):

$$
\varphi_{B 1}=\frac{F_{B 1}}{A_{B} f_{B} C_{B}}<1
$$

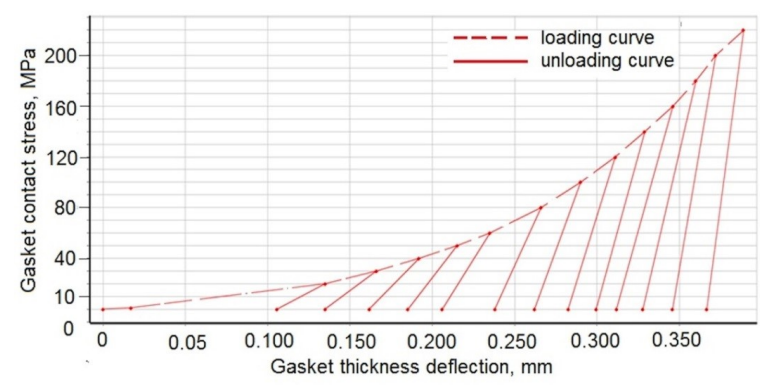

Figure 5: The characteristic of FG_1 gasket describing the deformation level as a function of contact stress

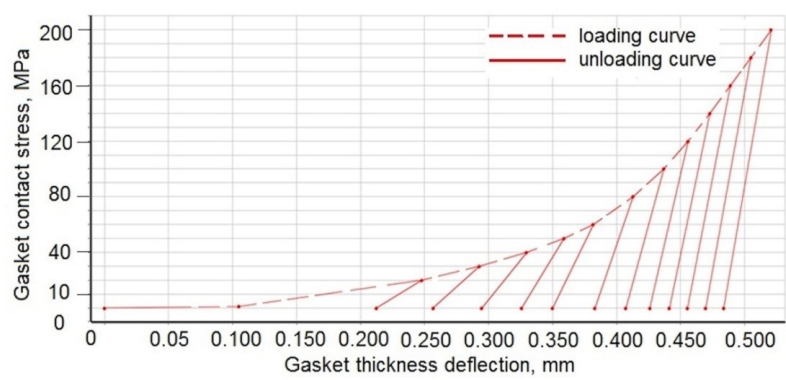

Figure 6: The characteristic of FG_2 gasket describing the deformation level as a function of contact stress

Gasket load ratio:

$$
\varphi_{G 1}=\frac{F_{G 1}}{A_{G} Q_{S \max }}<1
$$

Flange load ratio:

$$
\varphi_{F 1}=\frac{F_{G 1} \cdot h_{G}+F_{Q 1}\left(h_{H}-h_{P}\right)+F_{R 1} \cdot h_{H}}{W_{F}}
$$

Where:

$W_{F}$ - characteristic cross-section modulus of the flange [N-m] and $h_{G}, h_{H}, h_{P}$ - lever of the arms where particular forces are applied on characteristic points of the flange joint.

The calculation by means of a formula (6) is very complex, since it includes many parameters depending on the flange dimensions. In a simplified form the parameter $\Phi \mathrm{F}$ is a ratio between the actual stress of the flange to the allowable stress of the flange material.

Flange rotation is calculated as follows:

$$
\Theta_{F}=\frac{Z_{F}}{E_{F}}\left\{F_{G} h_{G}+F_{Q}\left(h_{H}-h_{P}+h_{Q}\right)+F_{R}\left(h_{H}-h_{R}\right\}\right.
$$

In service torque:

$$
M_{B 0}=k_{B} F_{B 0}
$$

Where $k_{B}$ is a bolt coefficient consisting of a characteristic dimension of the bolt as well as friction coefficient of the thread. 
Selected results of the analytical calculations for both load states I-0 and I-1 were presented in Tables from 2 to 3.

Considering the data in tables, it can be concluded that for two load states the values of the safety factors were less than 1 , which means that the construction meets the key criterion (1). The most loaded element of the joint in both load conditions was the bolt, while the least loaded was the flange. The required maximum tightening torque was $221 \mathrm{Nm}$ and was calculated for the joint gasketed with material GF_1. It was a value ensuring the design tightness of $0.1 \mathrm{mg} / \mathrm{s} \cdot \mathrm{m}$. The conclusion from the calculations was that the FG_1 material required a higher contact stress to obtain the same tightness level as the FG_2 gasket. Figure 7 presents a comparison of the safety factors (calculated in-service $\mathbf{I - 0}$ and the operating condition $\mathbf{I - 1}$ ) of the flange joint gasket with both configurations. The values of the safety factor presented in the charts were limited to the load providing tightness class L0.1.

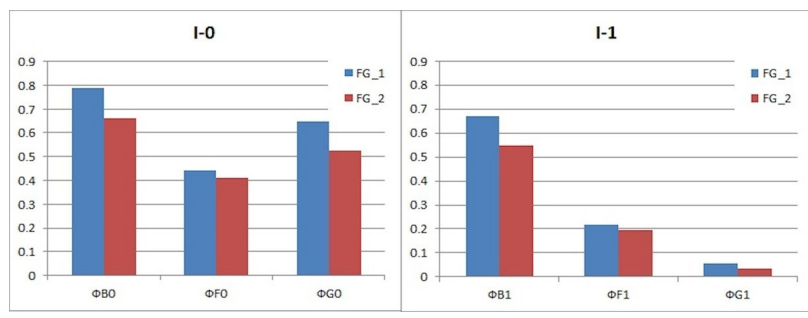

Figure 7: Safety factors in-service and operation states of the joint gasketed with two configurations -FG_1 and FG_2 materials

\section{Numerical calculations}

The main aim of the numerical calculations was to determine the local equivalent stress of particular elements of the joint as well as to determine the contact stress on the gasket surface.

\subsection{Computational model}

The numerical calculation were conducted in ANSYS 15.0 software using structural analysis. The geometric model of the joint and the boundary conditions were presented in Figure 8. On the lateral walls of $1 / 8$ th of flange bolted joint the frictionless supports were applied to get periodic model. To the bolt the pretension load was applied in order to simulate the bolt's force in service state. The internal surface of the joint was loaded by pressure to simulate the operational conditions. The calculations were carried out in two load steps; the first corresponded to the in-service state (I-0), the second one to the operating state resulting from the pressure of 40 bar. As the in-service load of the bolt, the values obtained from the analytical calculation corresponding to the design tightness class were applied (see the $F_{B 0}$ values included in Tables 2 and 3). On the mating surfaces the contact elements were activated as well as a friction coefficient 0.4 was set. Flanges, bolt, washer and a nut were mapped with an isotropic model of a steel material with Young's modulus $E=206000 \mathrm{MPa}$ and Poison ratio $\mathrm{v}=0.3$. The properties of the gasket as a numerical model were inserted to the computer program in the form of deformation characteristics, shown in Figures 5 and ??. Discretization of the model was carried out with hexahedra and wedge elements (see Fig. 9) with quadratic shape function. The contacts between particular items was modeled as a frictional. The friction coefficient between metallic part was 0.15 whereas the contact region between gasket and metal was 0.3 . a)

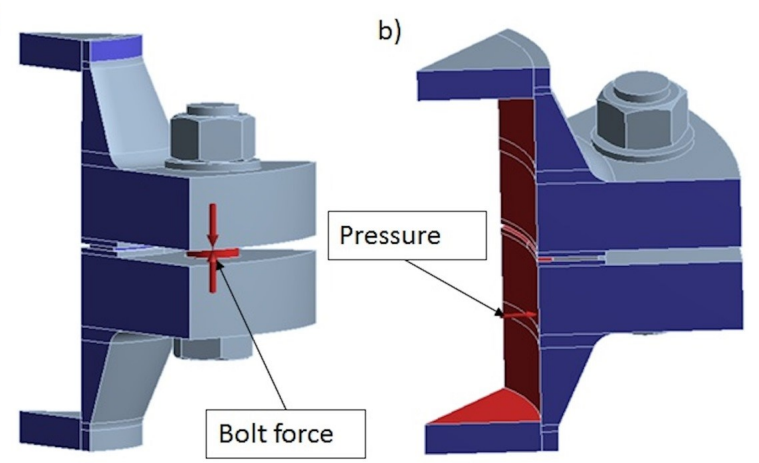

Figure 8: The geometrical model as well as boundary conditions of the joint: a) in service load $\mathbf{I}-\mathbf{0}$ b) operating load I-1 a)

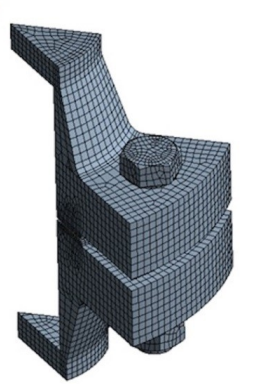

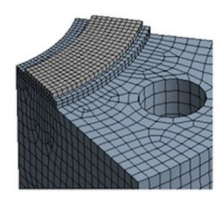

c)

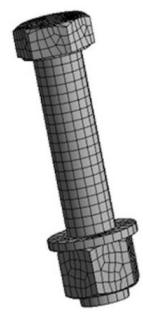

Figure 9: The computational mesh of the joint a) whole assembly, b) discretized region of the gasket and flange $\mathrm{c}$ ) fastener 
Table 2: The selected results of the flange-bolted PN40 DN 100 gasketed with material FG_1

\begin{tabular}{|c|c|c|c|c|c|c|c|c|c|c|c|}
\hline Design tightness class & \multicolumn{9}{|c|}{ I-0 } & \multicolumn{5}{c|}{ I-1 } \\
\cline { 2 - 11 } & $F_{B O}$ & $M_{B O}$ & $\Phi_{B O}$ & $\Phi_{F O}$ & $\Phi_{G O}$ & $\theta_{F O}$ & $F_{B 1}$ & $\Phi_{B 1}$ & $\Phi_{F 1}$ & $\Phi_{G 1}$ & $\theta_{F 1}$ \\
\hline & $\mathrm{kN}$ & $\mathrm{Nm}$ & - & - & - & 0 & $\mathrm{kN}$ & - & - & - & 0 \\
\hline $\mathrm{L} 1.0$ & $\mathbf{4 3 . 2}$ & $\mathbf{1 6 9}$ & 0.59 & 0.36 & 0.57 & 0.14 & 44.55 & 0.52 & 0.221 & 0.05 & 0.17 \\
\hline $\mathrm{L} 0.1$ & 56.4 & $\mathbf{2 2 1}$ & 0.79 & 0.44 & 0.65 & 0.18 & 58.38 & 0.67 & 0.219 & 0.058 & 0.22 \\
\hline
\end{tabular}

Table 3: The selected results of the flange-bolted PN40 DN 100 gasketed with material FG_2

\begin{tabular}{|c|c|c|c|c|c|c|c|c|c|c|c|}
\hline \multirow[t]{2}{*}{ Design tightness class } & \multicolumn{6}{|c|}{ I-O } & \multicolumn{5}{|c|}{ I-1 } \\
\hline & $F_{B O}$ & $M_{B O}$ & $\Phi_{B O}$ & $\Phi_{F O}$ & $\Phi_{G O}$ & $\theta_{F O}$ & $F_{B 1}$ & $\Phi_{B 1}$ & $\Phi_{F 1}$ & $\Phi_{G 1}$ & $\theta_{F 1}$ \\
\hline & $\mathrm{kN}$ & $\mathrm{Nm}$ & - & - & - & o & $\mathrm{kN}$ & - & - & - & $\circ$ \\
\hline L1.0 & 29.1 & 114 & 0.39 & 0.295 & 0.420 & 0.11 & 30.48 & 0.36 & 0.209 & 0.031 & 0.15 \\
\hline L0.1 & 46.6 & 182 & 0.66 & 0.409 & 0.525 & 0.14 & 48.37 & 0.55 & 0.197 & 0.035 & 0.19 \\
\hline
\end{tabular}

In the middle part of the gasket and flange rebate, measuring paths were introduced (see Fig. 10). After postprocessing the radial distribution of the contact stress on the gasket and the displacement of the flange were determined along these paths.
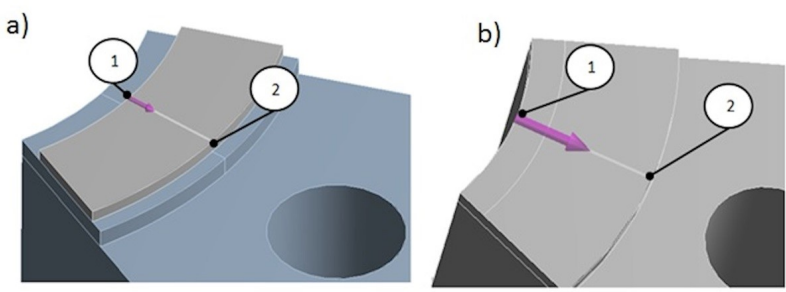

Figure 10: Defined measuring paths a) on the gasket b) on the flange

\subsection{Numerical results}

Based on the numerical calculation the following results were analyzed:

- distribution of the contact stress on the gasket surface,
- maximum equivalent stress in individual elements of the joint,

- increment of the bolts load resulting from the operating condition I-1,

- flange deformation (rotation angle of the flange in an axial plane of the joint).

\subsubsection{The distribution of the gasket contact stress}

Figures 11 and 12 present the maps of the contact stress distribution on the surface of the gasket in the operating state I-1. The unevenness of the contact stress along the radius of the gasket is caused by the deformation of the flange. In the load corresponding to tightness L1.0 in both joints, the contact stress difference from point 1 to point 2 was approximately $10 \mathrm{MPa}$. In case of the load corresponding to tightness L0.1, the pressure difference was about $12 \mathrm{MPa}$ for the joint gasketed with material $\mathrm{FG}_{-}$, whereas in the joint gasketed with FG_1 the difference was $20 \mathrm{MPa}$. A more accurate plot of the contact stress variation along the radial path of the gasket was shown in Figure 13.

On the chart, the radial contact stress of the joint gasketed with FG_1 and FG_2 materials in the operating state 

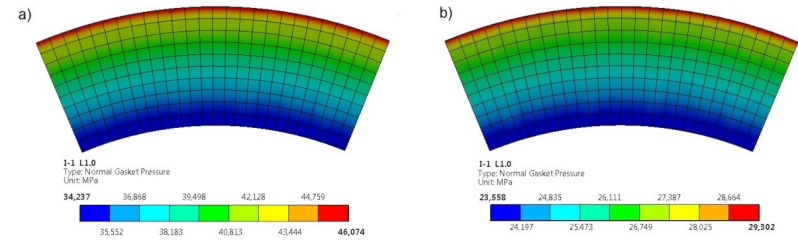

Figure 11: Contact stress distribution in operating state and flange load corresponding to tightness L1.0 a) joint gasketed with FG_1 b) joint gasketed FG_2

I-1 and load corresponding to the class L0.1 were compared. While analyzing these data it can be noted that the joint gasketed with FG_1 is characterized by a greater nonuniformity of the radial contact stress than the joint gasketed with material FG_2 (compare the slope of the contact stress of both curves presented in Fig. 13). Figures 14 and 15 present a comparison of the radial contact stress in both load states I-0 and I-1.
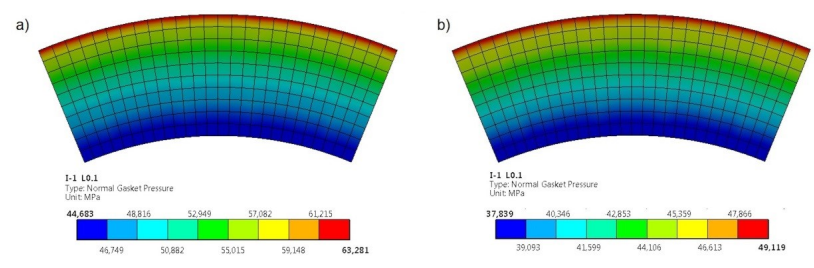

Figure 12: Contact stress distribution in operating state and flange load corresponding to tightness L0.1 a) joint gasketed with FG_1 b) joint gasketed FG_2

The dashed lines in the diagrams correspond to the in-service state (I-0) and the continuous lines to the operating state (I-1). The pressure difference in I-0 and I-1 states resulted from the partial unloading of the gasket due to the hydrostatic pressure acting on the blinded ends of flanges. In both cases of joint configurations, regardless of the tightness level, the differences of the contact stress between states I- 0 and I- 1 were the same and amounted to about $5 \mathrm{MPa}$.

\subsubsection{Maximum stress}

Fig. 16 presents examples of equivalent stress distribution in the root of the bolt and the most stressed fragment of the flange.

The maximum local stress of the joint in particular load states were referred to the permissible design stress, as a result the safety ratios were calculated in line with the formula (1). The results of the calculations were presented in tables 4 and 5 . All calculated values of the safety factors

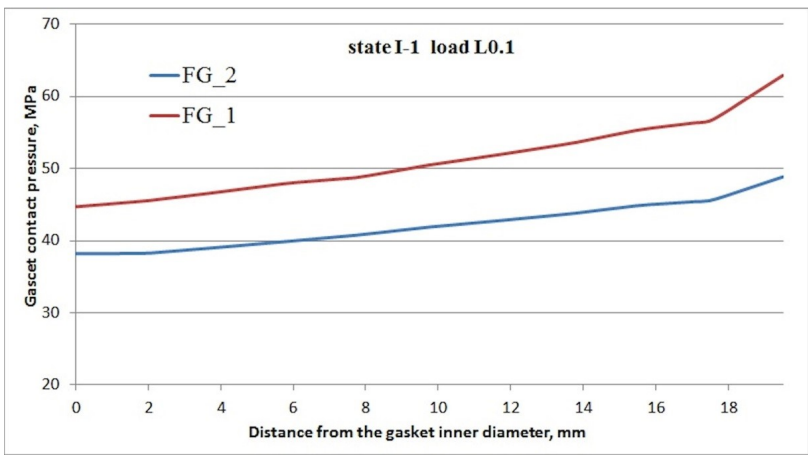

Figure 13: The radial contact stress along the gasket width in case of joint load corresponding to tightness L0.1

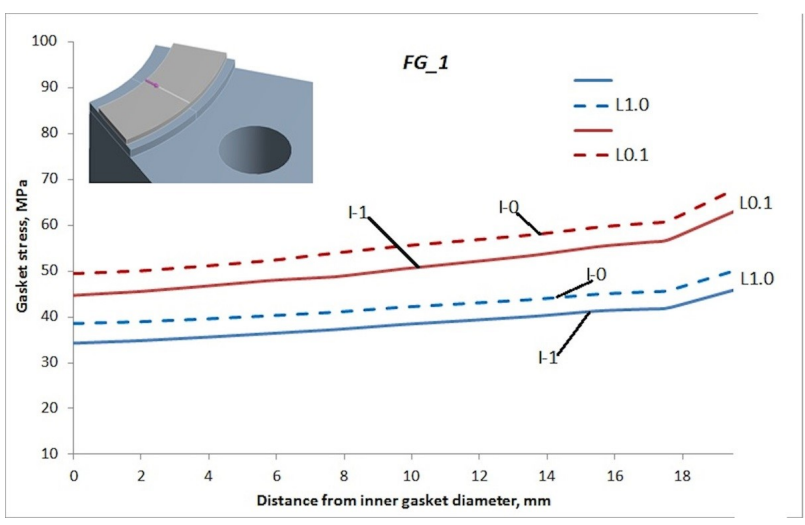

Figure 14: The radial contact stress along the gasket width in joint gasketed with FG_1 in all cases of loads

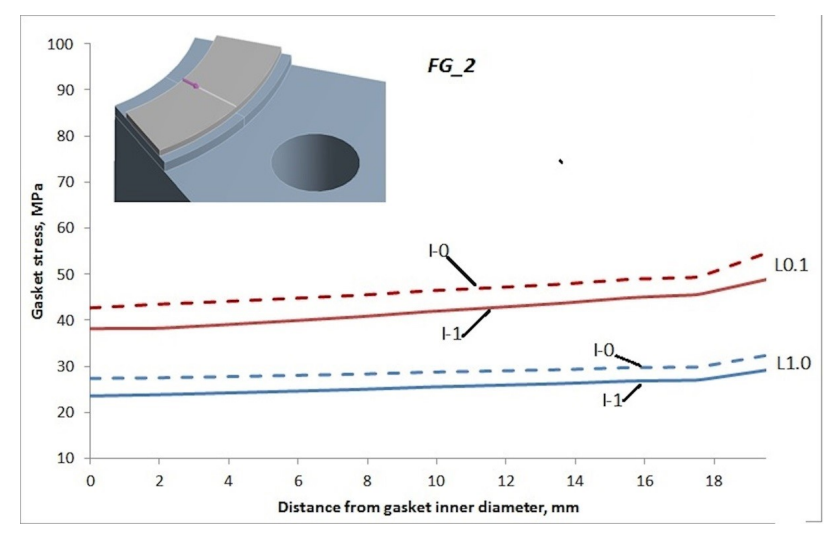

Figure 15: The radial contact stress along the gasket width in joint gasketed with FG_2 in all cases of loads

were in the acceptable range. Moreover, the tables contain the load increase in the bolt and the rotation angle of the flange. The load increase of the bolts in I-1 state equals, on average, $1 \mathrm{kN}$ and is the result of the hydrostatic force in line with the formula (3). To better illustrate the results of the calculations in figure 17 the bar charts of the values of safety factors and rotation angle of the flange against 


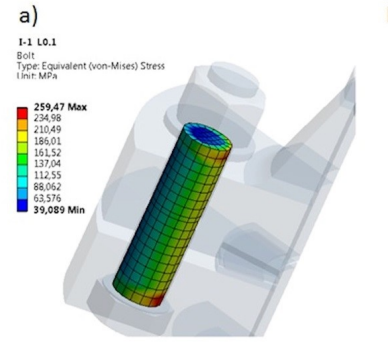

b)

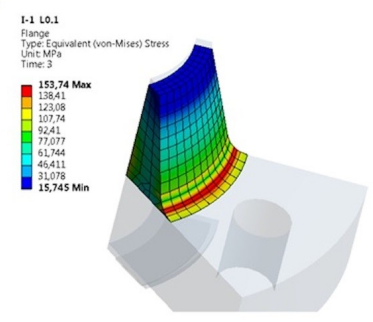

Figure 16: Equivalent stress distribution in the joint a) in root of the bolt b) in flange

the background of the results obtained analytically were presented. The values in the charts refer to the joint load corresponding to L0.1 tightness.

Studying the chart, data shows that, according to the numerical calculations the flange was the most loaded element $\Phi \mathrm{F}=0.8$. However, as per the analytical calculations the most loaded element is the bolt $\Phi \mathrm{B}=0.68$. Furthermore, considering the analytical calculations the safety factor of the gasket is below $\Phi \mathrm{G}=0.1$, and as per the numerical calculations, the value is above $\Phi \mathrm{G}=0.25$. Nevertheless, regardless of the method of calculations, all safety factors (according to the formula (1)) meet the basic requirement. The largest differences between the numerical and analytical data were obtained for the rotation angle of the flange. The values of this parameter, according to analytical calculations, were four times higher than the results obtained by the numerical calculations.

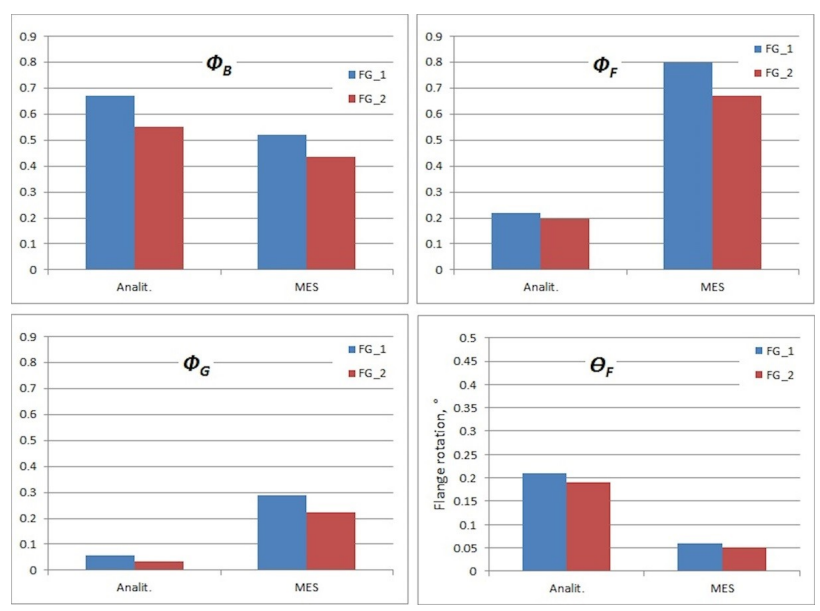

Figure 17: Graphical representation of safety factors in joint elements and rotation angles of the flanges of the joint gasketed with FG_1 and FG_2. State I-1and joint load corresponding to tightness L0.1, a) bolt safety factors, b) flange safety factors, c) gasket safety factors, d) rotation angle of the flange.

\section{Experimental tests}

Verifications of the analytical and numerical results were performed by means of the experimental tests. The main goal was to confirm the assumed (design) tightness as well as to verify the forces obtained from the calculations, tightening torques of the nuts and estimated safety factors of the bolt and gasket.

\subsection{Test rig}

Fig. 18 presents a test rig whose main part was the flangebolted joint with dimensions corresponding to Table 1 . Additional equipment of the test rig was: amplifier associated with a system of strain gauges fixed to each of the eight bolts M 24, a helium detector together with a vacuum pump, a helium reservoir with a pressure of 200 bar. Due to the fact that each bolt was equipped with a strain gauge system it was possible to determine their exact tension under a given load caused by a torque wrench.

\subsection{Test procedure}

Before mounting the upper flange (in order to determine the contact stress), two fragments of a FUJI measuring film with a range of 10-50 $\mathrm{MPa}$ (medium stress) and a range of 50 to $130 \mathrm{MPa}$ (high stress) were placed on the gasket surface (see Fig. 18c). Then, after placing the upper flange, the bolts were installed in the holes of flanges and pre-tighten the nuts. The test procedure was carried out in two main steps. In the first step (corresponding to state I-0), the joint was loaded by tightening the nuts with a torque wrench in accordance with the values included in Tables 2 and 3 (see values of $M_{B 0}$ for two gaskets). Next, the values of the strain gauges of each bolt were recorded. In the second step (corresponding to state I-1), the joint was loaded with 40 bar helium pressure. As a result the joint was pressurized, the values of the bolt tension were read again and the level of helium leakage was measured. After completing the measurements, the joint was decompressed, the nuts unscrewed and the upper flange removed. An indirect step in the measurements was to determine the contact stress on the gasket surface. As mentioned above, the measurement was performed by means of the FUJI measuring film. The principle of its work is based on the fact that under the contact stress the film was colored in pink. If the contact stress was greater, the color of the film was more intensive. To obtain the value of the contact stress the intensity of the film was evaluated on the basis of the reference scale. 
Table 4: The results of numerical calculation of the joint gasketed with FG_1 material

\begin{tabular}{|c|c|c|c|c|c|c|c|c|c|c|}
\hline & \multicolumn{5}{|c|}{ I-0 } & \multicolumn{5}{c|}{ I-1 } \\
\cline { 2 - 11 } & $F_{B O}$ & $\Phi_{B O}$ & $\Phi_{F 0}$ & $\Phi_{G O}$ & $\theta_{F O}$ & $F_{B 1}$ & $\Phi_{B 1}$ & $\Phi_{F 1}$ & $\Phi_{G 1}$ & $\theta_{F 1}$ \\
\hline $\mathrm{L} 1.0$ & $\mathbf{4 3 2 0 0}$ & 0.391 & 0.599 & 0.229 & 0.04 & 44363 & 0.402 & 0.615 & 0.209 & 0.05 \\
\hline $\mathrm{L} 0.1$ & $\mathbf{5 6 4 2 9}$ & 0.510 & 0.788 & 0.309 & 0.06 & 57286 & 0.519 & 0.801 & 0.288 & 0.06 \\
\hline
\end{tabular}

Table 5: The results of numerical calculation of the joint gasketed with FG_2 material

\begin{tabular}{|c|c|c|c|c|c|c|c|c|c|c|}
\hline & \multicolumn{9}{|c|}{ I-0 } & \multicolumn{5}{c|}{ I-1 } \\
\cline { 2 - 10 } & $F_{B O}$ & $\Phi_{B O}$ & $\Phi_{F O}$ & $\Phi_{G O}$ & $\theta_{F O}$ & $F_{B 1}$ & $\Phi_{B 1}$ & $\Phi_{F 1}$ & $\Phi_{G 1}$ & $\theta_{F 1}$ \\
\hline L1.0 & 29085 & 0.263 & 0.407 & 0.148 & 0.03 & 31077 & 0.282 & 0.437 & 0.133 & 0.03 \\
\hline L0.1 & 46586 & 0.421 & 0.652 & 0.249 & 0.05 & 47957 & 0.435 & 0.671 & 0.223 & 0.05 \\
\hline
\end{tabular}

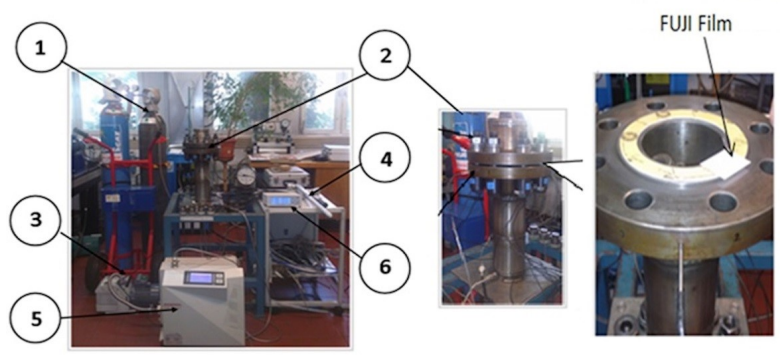

Figure 18: Test rig to investigate flange-bolted joint with gasket 1 -helium reservoir, 2 - flange-bolted joint, 3 - vacuum pump, 4 torque wrench, 5 - helium detector, 6 - amplifier

\subsection{Test results}

The Tables 6 and 7 present the forces of the bolts in individual load conditions, the average contact stress and the measured values of the leakage. The most important value obtained from The experimental test was the value of helium leakage. In case of a joint load corresponding to the design tightness L0.1 the real (measured) values of the leakage were in an acceptable order for both configurations of the joint. It means that analytically calculated values of torque $\mathrm{M}_{B 0}$ were correct The greatest discrepancy of the real leakage, in respect to the design value, was measured in case of the joint load corresponding to the design tightness L0.1. Nonetheless, the measured tightness was higher than the design values. It means that the calculated torque in this case was slightly excessive.

The increment in the force of bolts due to helium pressure acting on the blinded ends of the flanges (in both joint configurations) approximately equals $1 \mathrm{kN}$. These values are consistent with the load increment resulting from the numerical calculations. The average gasket contact stress in I-0 state was calculated according to the formula (9), whereas in I-1 state according to the formula (10).

$$
\begin{gathered}
S_{0}=\frac{8 \bar{F}_{B 0}}{A_{G}} \\
S_{1}=\frac{\left(8 \bar{F}_{B 1}-p A_{I}\right)}{A_{G}}
\end{gathered}
$$

On the basis of the average force in the bolt and the values of the average contact stress of the gasket, the safety factors were calculated. In Fig. 19 the values of safety factors were listed in the form of a bar chart. The analysis of these data shows that the bolt was a more loaded element.

In state I- 0 the safety factor of the bolt was $\Phi \mathrm{B}=0.37$ for the joint gasketed with FG_1, while in the joint gasketed with $\mathrm{FG} \_2$ the value was $\Phi \mathrm{B}=0.31$. In case of the gasket load these values were $\Phi \mathrm{G}=0.26$ and $\Phi \mathrm{G}=0.23$, respectively. Fig. 20 presents an example of the contact stress distribution obtained with the FUJI measuring film.

Using the FUJI film color palette the contact stress was estimated at three characteristic points of the gasket, 
Table 6: Listing of measurement and target data in the form of tightening torque of nuts, tightness class and bolt forces for joint gasketed FG_1 material.

\begin{tabular}{|c|c|c|c|c|c|c|c|c|c|c|c|}
\hline \multicolumn{12}{|c|}{ I-0 } \\
\hline Ldesign & Mc & $\mathbf{F}_{1}$ & $F_{2}$ & $F_{3}$ & $F_{4}$ & $F_{5}$ & $F_{6}$ & $\mathbf{F}_{7}$ & $F_{8}$ & $F_{\text {śr }}$ & $\mathbf{S}_{0}$ \\
\hline $\mathrm{mg} / \mathrm{s} \cdot \mathrm{m}$ & $\mathrm{Nm}$ & $\mathrm{kN}$ & $\mathrm{kN}$ & $\mathrm{kN}$ & $\mathrm{kN}$ & $\mathrm{kN}$ & $\mathrm{kN}$ & $\mathrm{kN}$ & $\mathrm{kN}$ & $\mathrm{kN}$ & $\mathrm{MPa}$ \\
\hline 1 & 169 & 44.54 & 45.32 & 47.83 & 47.15 & 47.43 & 47.24 & 45.92 & 48.96 & 46.80 & 45.14 \\
\hline 0.1 & 221 & 53.99 & 56.64 & 59.8 & 59.35 & 59.23 & 59.9 & 54.09 & 60.28 & 57.91 & 55.86 \\
\hline \multicolumn{12}{|c|}{ I-1 } \\
\hline $\mathbf{L}_{\text {design }}$ & $\mathbf{L}_{\text {real }}$ & $\mathbf{F}_{1}$ & $\mathbf{F}_{2}$ & $F_{3}$ & $F_{4}$ & $F_{5}$ & $F_{6}$ & $\mathbf{F}_{7}$ & $F_{8}$ & $F_{\text {sr }}$ & $\mathbf{S}_{\mathbf{I}}$ \\
\hline $\mathrm{mg} / \mathrm{s} \cdot \mathrm{m}$ & $\mathrm{mg} / \mathrm{s}^{\star} \mathrm{m}$ & $\mathrm{kN}$ & $\mathrm{kN}$ & $\mathrm{kN}$ & $\mathrm{kN}$ & $\mathrm{kN}$ & $\mathrm{kN}$ & $\mathrm{kN}$ & $\mathrm{kN}$ & $\mathrm{kN}$ & $\mathrm{MPa}$ \\
\hline 1 & 4.88 & 44.84 & 45.62 & 48.13 & 47.45 & 47.73 & 47.54 & 46.22 & 49.26 & 47.10 & 40.68 \\
\hline 0.1 & 0.29 & 54.09 & 56.74 & 59.9 & 59.45 & 59.33 & 60 & 54.19 & 60.38 & 58.01 & 51.20 \\
\hline
\end{tabular}

Table 7: Listing of measurement and target data in the form of tightening torque of nuts, tightness class and bolt forces for joint gasketed FG_2 material.

\begin{tabular}{|c|c|c|c|c|c|c|c|c|c|c|c|}
\hline \multicolumn{12}{|c|}{ I-0 } \\
\hline $\mathbf{L}_{\text {design }}$ & Mc & $F_{1}$ & $F_{2}$ & $F_{3}$ & $F_{4}$ & $F_{5}$ & $F_{6}$ & $F_{7}$ & $F_{8}$ & $F_{s ́ r}$ & $\mathbf{S}_{0}$ \\
\hline $\mathrm{mg} / \mathrm{s} \bullet \mathrm{m}$ & $\mathrm{Nm}$ & $\mathrm{kN}$ & $\mathrm{kN}$ & $\mathrm{kN}$ & $\mathrm{kN}$ & $\mathrm{kN}$ & $\mathrm{kN}$ & $\mathrm{kN}$ & $\mathrm{kN}$ & $\mathrm{kN}$ & $\mathrm{MPa}$ \\
\hline 1 & 114 & 29.99 & 30.78 & 31.18 & 32.73 & 31.04 & 30.91 & 29.33 & 32.3 & 27.00 & 26.04 \\
\hline 0.1 & 182 & 46.19 & 47.71 & 49.14 & 49.35 & 48.59 & 49.9 & 47.09 & 50.28 & 48.53 & 46.81 \\
\hline \multicolumn{12}{|c|}{ I-1 } \\
\hline$L_{\text {design }}$ & $\mathrm{L}_{\text {real }}$ & $F_{1}$ & $F_{2}$ & $F_{3}$ & $F_{4}$ & $F_{5}$ & $F_{6}$ & $F_{7}$ & $F_{8}$ & $F_{\text {śr }}$ & $\mathbf{S}_{\mathrm{I}}$ \\
\hline $\mathrm{mg} / \mathrm{s} \bullet \mathrm{m}$ & $\mathrm{mg} / \mathrm{s} \bullet \mathrm{m}$ & $\mathrm{kN}$ & $\mathrm{kN}$ & $\mathrm{kN}$ & $\mathrm{kN}$ & $\mathrm{kN}$ & $\mathrm{kN}$ & $\mathrm{kN}$ & $\mathrm{kN}$ & $\mathrm{kN}$ & Mpa \\
\hline 1 & 2.66 & 30.99 & 31.78 & 32.18 & 33.73 & 32.04 & 31.91 & 30.33 & 33.3 & 27.87 & 22.13 \\
\hline 0.1 & 0.39 & 47.09 & 48.61 & 50.04 & 50.25 & 49.49 & 50.8 & 47.99 & 51.18 & 49.43 & 42.93 \\
\hline
\end{tabular}




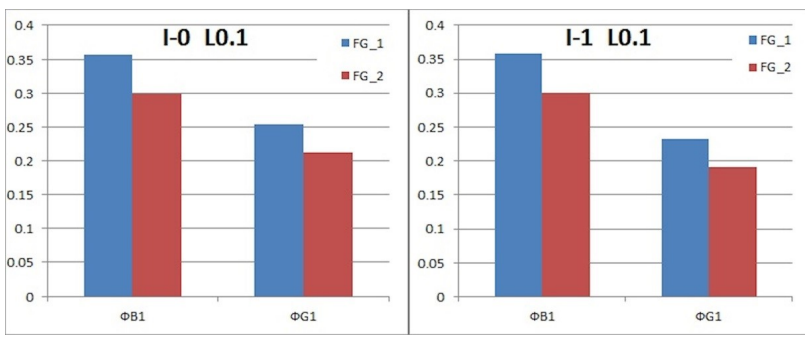

Figure 19: The safety factors of the bolt and gasket in case of joint load corresponding to tightness L0.1

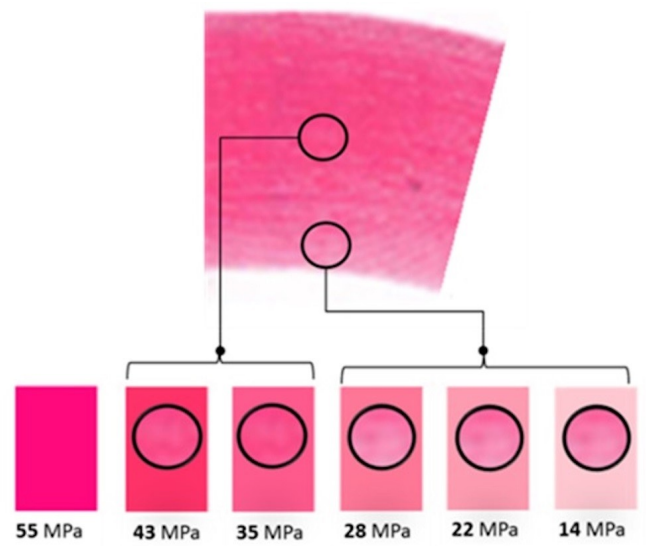

Figure 20: Contact stress distributions on the FUJI film in case of joint gasketed with FG_2 material and state I-0 as well as load corresponding to tightness $\mathrm{L1} .0$

i.e. on the inner, mean and outer diameter. According to Fig. 20, the contact stress on the inner and outer diameter matched better a reference color $28 \mathrm{MPa}$. In case of the mean diameter, the film color matched most the reference value of $35 \mathrm{MPa}$.

\section{Summary of the test and calculations}

The results obtained by experimental tests confirm that the proposed method of the analytical calculations allows to achieve the assumed level of the joint tightness with sufficient accuracy.

The calculated values of torque in a satisfactory way provide a proper bolt tension (compare $\mathrm{F}_{B 0}$ data calculated analytically and determined experimentally). Moreover, these values provide the assumed degree of the joint tightness. In addition, it was found that the joint is optimally loaded as proved by the calculated and verified values of the safety factors.

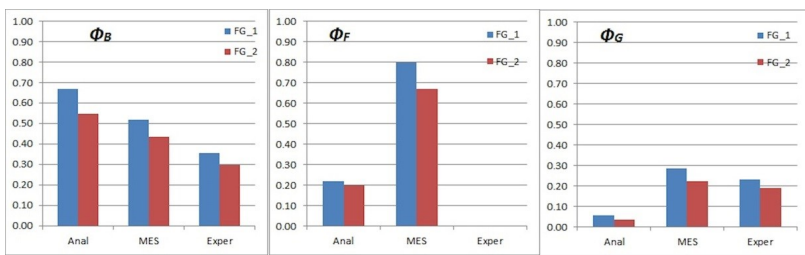

Figure 21: Listing of the safety factors calculated analytically and numerically as well as experimentally determined
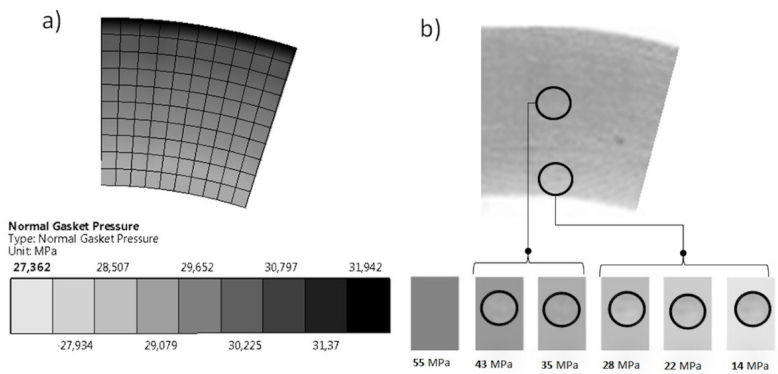

Figure 22: The comparison of numerical and test results of the contact stress distribution on the gasket FG_2 in state I-0 corresponding to tightness L1.0

Figure 21 presents a comparison of the safety factors calculated analytically and numerically as well as those determined experimentally. Each value was under a permissible level in line with the criterion (1). Another aspect worthy comparison is the numerically calculated and experimentally determined distribution of the contact stress. The comparison of both results was presented in Fig. 22. For a better comparison of both results, the map was presented in a gray scale. It can be seen that the range of values in both cases is counted together. In case of the numerical result, the contact stress ranged from 27 to $32 \mathrm{MPa}$, whereas from the map of the FUJI film the range was from 28 to $35 \mathrm{MPa}$. The only contradiction between the contact stress determined by the FUJI film and numerically obtained is the fact that in case of the FUJI film the contact stress on the outer diameter reduces which does not comply with the numerical results.

\section{References}

[1] EN 1591-1. Flanges and their joints - Design rules for gasketed circular flange connections - Part 3: Calculation. 2014

[2] ESA-FSA "Guidelines for safe seals usage -Flange and Gasket" Part 1: Guidelines for maintenance,

[3] EN 1591-4 Qualification of personnel competency in the assembly of the bolted connections of critical service pressurized systems. 2014 
[4] Kidam K., Hassin M. H. „Enhancement of inherent safety and accident prevention In chemical industry by preventing past accidents, Journal of Chemical and Natural Resources Engineering 2008 pp 75-86

[5] Sonnemans P.J.M., Korvers P.M.W „Accidents in the chemical industry" Journal of Loss Preventation in the Process Industry, 2006, pp. 1-12

[6] Furtek A. „Review of hazardous of the pipeline accidents” Centre of Excellence at the Institute of Atomic Energy, 2004,

[7] TA Luft "First general administrative regulation to the federal emission protection law -technical guideline for clean air " July 24, 2002

[8] ASME Boiler and Pressure Vessel Code. Section VIII. Division 1. ASME. 2017

[9] PN-EN 13445-3 Unfired pressure vessels- Part 3: Design. 201411/A4:2018

[10] EN 13555. Flanges and their joints - Gasket parameters and test procedures relevant to the design rules for gasketed circular flange connection. 2014.

[11] Abid M. "Assembly of gasketed bolted flange joints using torque control of preload method: FEM approach". ASME 2013 Pressure Vessels and Piping Conference. Volume 3: Design and Analysis. Paris. France. July 14-18. 2013.

[12] Estrada H.. Parsons I. D. „Strength and leakage finite element analysis of a GFRP flange joint". International Journal of Pressure Vessel and Piping. 76. 1999. 543-550.
[13] Joshi D. Mahadevan P. „Unimportance of geometric nonlinearity in analysis of flanged joint with metal-to-metal contact”. International Journal of Pressure Vessels and Piping 84 (2007) 405411.

[14] Murali M.. Shunmugam M.S.. Siva N. „A study on the sealing performance of bolted flange joint with gaskets using finite element analysis" International Journal of Pressure Vessels and Piping 84 (2007) 349-357.

[15] Walczak R., Pawlicki J, Zagórski A., Tightness and material aspects of bolted flange connections with gaskets of nonlinear, Arch. Metall. Mater., Vol. 61 (2016), No 3, p. 1409-1416.

[16] Grzejda R., Impact of Nonlinearity of the Contact Layer Between Elements Joined in a Preloaded Bolted Flange Joint on Operational Forces in the Bolts, Mechanics and Mechanical Engineering Vol. 21, No. 3 (2017) p.541-548.

[17] Sawa T., Tenma K., Kobayashi T., Kurosawa R., Finite Element Method Stress Analysis and Evaluation of the Sealing Performance in Box-Shape Flange Gasketed Connections Subjected to Internal Pressure, J. Press. Vessel Technol., vol. 139, no. 5, p. 051202 , Aug. 2017.

[18] Cao D.. Xu H. "3-D Finite Element Analysis of Bolted Flange Joint considering gasket non-linearity". ASME Pressure Vessels and Piping. Vol 382. 1999 pp 121-126. 